\title{
Enhancing Multiple Domains of Well-Being by Decreasing Multiple Health Risk Behaviors: A Randomized Clinical Trial
}

\author{
James O. Prochaska, PhD, Kerry E. Evers, $\mathrm{PhD},{ }^{2}$ Patricia H. Castle, $\mathrm{PhD}$, ${ }^{2}$ Janet L. Johnson, $\mathrm{PhD},{ }^{2}$ \\ Janice M. Prochaska, PhD, ${ }^{2}$ Elizabeth Y. Rula, PhD, ${ }^{3}$ Carter Coberley, PhD, and James E. Pope, MD ${ }^{3}$
}

\begin{abstract}
Tailored behavior change programs have proven effective at decreasing health risk factors, but the impact of such programs on participant well-being has not been tested. This randomized trial evaluated the impact of tailored telephone coaching and Internet interventions on health risk behaviors and individual well-being. Exercise and stress management were the primary health risks of interest; improvements in other health risk behaviors were secondary outcomes. A sample of 3391 individuals who reported health risk in the areas of exercise and stress management were randomly assigned to 3 groups: telephonic coaching that applied Transtheoretical Model (TTM) tailoring for exercise and minimal tailoring (stage of change) for stress management; an Internet program that applied TTM tailoring for stress management and minimal tailoring for exercise; or a control group that received an assessment only. Participants were administered the Well-Being Assessment and, at baseline, had relatively low well-being scores (mean, 60.9 out of 100 across all groups). At 6 months, a significantly higher percentage of both treatment groups progressed to the Action stage for exercise, stress management, healthy diet, and total number of health risks, compared to the control group. Both treatment groups also demonstrated significantly greater improvements on overall well-being and the domains of emotional health, physical health, life evaluation, and healthy behaviors. There were no differences between the groups for 2 well-being domains: basic access to needs and work environment. These results indicate that scalable, tailored behavior change programs can effectively reduce health risk and accrue to improved wellbeing for participants. (Population Health Management 2012;15:276-286)
\end{abstract}

\section{Introduction}

$\mathbf{U}$ NHEALTHY BEHAVIORS are well-established risk factors for poor health outcomes, primarily as a result of the increased likelihood of chronic disease imparted by unhealthy lifestyle behaviors. The importance of promoting healthy behaviors as a means to reduce disease burden and associated morbidity and health care costs is well established. In 1979, the Surgeon General released the first Healthy People report on health promotion and disease prevention objectives in the United States. ${ }^{1}$ This report, updated every decade, included for the first time in Healthy People 2020 an objective to improve the well-being of Americans. ${ }^{1}$ The growing emphasis on enhancing well-being is based, in part, on a more inclusive definition of health, like that used by the World Health Organization that defines health as "a state of complete physical, mental, and social well-being and not merely the absence of disease or infirmity. ${ }^{2}$ Wellness programs have been aimed primarily at preventing infirmity by reducing health risk behaviors; however, in achieving this goal, such programs may also serve to improve the well-being of participants. This study is the first randomized trial to evaluate multiple domains of well-being (physical health, emotional health, healthy behaviors, life evaluation, work environment, and basic access) as more inclusive outcomes generated by high-impact wellness programs.

Each of the well-being domains captured in the Healthways Well-Being Assessment (WBA), have been found to be related to independent indicators of health, happiness, quality of life, functioning, health care costs, and/or lost productivity. Self-reports of physical health problems, for example, have been found to be related to health care costs, quality of life, and lost productivity resulting from absenteeism and presenteeism. ${ }^{3-6}$ Self-reports of emotional health

\footnotetext{
${ }^{1}$ Cancer Prevention Research Center, University of Rhode Island, Kingston, Rhode Island.

${ }^{2}$ Pro-Change Behavior Systems, Inc., West Kingston, Rhode Island.

${ }^{3}$ Center for Health Research, Healthways, Franklin, Tennessee.
} 
problems are related to the presence of depression, anxiety, and other mental health problems, decreased quality of life, and lost productivity. ${ }^{6-8}$ Conversely, higher levels of happiness have been found to be related to less mental and physical illness, better social relationships, helping others at work, and higher economic well-being. ${ }^{9}$ Healthy behaviors are related to decreased risk of chronic disease, disability, and mortality, as well as lower health care costs and presenteeism. ${ }^{10,11}$ A positive work environment predicts greater happiness, less stress, better social relationships, and greater productivity. ${ }^{12,13}$ Inadequate access to basic resources is strongly related to poverty levels, which is an independent predictor of increased morbidity, disability, and premature mortality. ${ }^{14,15}$ Finally, life satisfaction is related to physical and emotional health, higher quality of life, lower behavior risks, and greater productivity. ${ }^{16}$

In spite of the large body of research demonstrating associations between the fundamental components of wellbeing and health, health care costs, productivity, and other economic indicators, we could not identify any large randomized controlled trials (RCTs) that measured the impact of interventions on multiple domains of well-being. Support for such a study is provided by important smaller studies testing the impact of program interventions on single wellbeing domains or variables, such as happiness ${ }^{17}$ or positivity. ${ }^{18}$ There is also evidence that workplace wellness programs are associated with higher well-being in the domains of physical health, emotional health, and healthy behaviors. ${ }^{19}$ Together, this prior work demonstrates the potential for well-being domains to change as a result of intervention.

Although healthy behavior is included as a domain of the WBA, and thus relates to overall well-being, there is strong evidence from a previous study that health risk behaviors have consistent relationships with other domains of wellbeing. ${ }^{20}$ Both present life evaluation and future life evaluation were linearly related to the number of health risk behaviors, where present and future life evaluation declined as the number of risk behaviors increased. Similarly, physical and emotional health ladder ratings were linearly related to the number of health risk behaviors, with lower health ratings as the number of multiple risk behaviors increased. ${ }^{20}$ The results from our prior study suggested that evidencebased health behavior change programs used for wellness and disease management have the potential to enhance multiple dimensions of well-being. Therefore, participants in such programs may benefit to a greater extent than captured in traditional outcomes evaluations that focus only on selfreported or objective health risk data.

The wellness interventions tested in this trial were built upon research that has demonstrated that simultaneously treating populations with interventions tailored on the Transtheoretical Model (TTM) for multiple behaviors can be effective with each behavior. ${ }^{21-24}$ What has not been tested is whether such interventions can simultaneously reduce multiple behavior risk factors and increase multiple domains of well-being. Although a variety of behaviors relate to wellbeing, ${ }^{20}$ exercise and effective stress management were chosen as the 2 health behaviors that would have the greatest potential for changing multiple domains of well-being. Exercise has been found to produce over 60 different benefits, including reduction of depression, effective management of stress, decreased pain, increased self-esteem, better sleep, greater energy, and productivity. ${ }^{25}$ In addition, effective stress management has been found to decrease stress and depression, improve sleep, decrease pain, and increase productivity. ${ }^{26}$

The aim of this study was to evaluate the impact of 2 interventions; one employing full TTM tailoring online for the primary behavior of stress management and the other employing optimal TTM tailoring with coaches for the primary behavior of exercise. Both interventions employed stage matched feedback for the alternative behavior (considered secondary). These programs differ in a number of ways, but were applied because they are available services that we believed had the potential to reduce multiple risk behaviors and increase multiple domains of well-being. The authors hypothesized that the programs would help participants progress to the action stage on multiple health risk behaviors and would enhance overall well-being by increasing levels of well-being in 4 domains: healthy behaviors, physical health, emotional health, and life evaluation.

\section{Methods}

\section{Study design}

This RCT included 3 arms: (1) Telephonic coaching with exercise as the primary behavioral target and stress management as secondary; (2) Online program with stress management as the primary and exercise as the secondary behavior; and (3) Control group, which received assessments at baseline and 6-month follow-up, but no treatment.

\section{Participants}

Participants were recruited via the Internet through a survey sampling company that has a national panel of about $1,500,000$ potential participants. Panel members were invited to participate through the company by e-mail. Interested individuals then completed a short screening survey to ensure that eligibility requirements were met, including access to a computer and English speaking. In addition, to be eligible, participants had to report risk in the areas of both exercise (not engaging in moderate or vigorous exercise to guidelines) and stress (reporting stress that was not being managed effectively). Individuals who met screening criteria were then linked to the study consent materials before they were able to continue. The survey company was no longer involved in the study at this point except for the delivery of incentives. Each participant had a unique ID and was able to create an individual password. The computer system directed each individual to their next task throughout the study based on their login information. However, a support e-mail and 1-800 number were available for those who had any questions.

A total of 3391 individuals who consented to participate in the study were randomized to one of the 3 treatment conditions and immediately completed an online baseline assessment. Tables 1 and 2 present the demographics, body mass index, smoking history, and stage of change for exercise and effective stress management. Although all participants initially reported risk in the areas of stress or exercise, some participants indicated not being at risk (being in the Action or Maintenance stage of change for stress and exercise) in the baseline assessment. 
Table 1. Participant Baseline Demographics

\begin{tabular}{|c|c|c|c|c|}
\hline & $\begin{array}{l}\text { Group } 1 \\
\text { Control }\end{array}$ & $\begin{array}{c}\text { Group } 2 \\
\text { Stress Online }\end{array}$ & $\begin{array}{c}\text { Group } 3 \\
\text { Exercise Coaching }\end{array}$ & Total (1-3) \\
\hline \multicolumn{5}{|l|}{ Sex } \\
\hline Female & $59.6 \%(618)$ & $57.5 \%(746)$ & $59.2 \%(626)$ & $58.77 \%(1675)$ \\
\hline \multicolumn{5}{|l|}{ Ethnicity } \\
\hline White, not Hispanic & $81.7 \%$ (847) & $77.9 \%$ (1011) & $75.2 \%(795)$ & $78.27 \%$ (2653) \\
\hline Black, not Hispanic & $8.5 \%(88)$ & $11.3 \%(147)$ & $12.7 \%(134)$ & $10.83 \%(369)$ \\
\hline Asian/Other Pacific Islander & $1.7 \%(18)$ & $1.6 \%(21)$ & $1.2 \%(13)$ & $1.50 \%(52)$ \\
\hline American Indian or Alaskan Native & $0.4 \%(4)$ & $0.5 \%(7)$ & $0.3 \%(3)$ & $0.40 \%(14)$ \\
\hline Hispanic & $2.5 \%(26)$ & $3.4 \%(44)$ & $3.6 \%(38)$ & $3.17 \%(108)$ \\
\hline Other & $4.1 \%(43)$ & $3.4 \%(44)$ & $5.0 \%(53)$ & $4.17 \%(140)$ \\
\hline Combination & $1.1 \%(11)$ & $1.8 \%(23)$ & $2.0 \%(21)$ & $1.63 \%(55)$ \\
\hline \multicolumn{5}{|l|}{ Currently employed } \\
\hline No & $47.4 \%(492)$ & $48.2 \%(625)$ & $47.7 \%(504)$ & $47.77 \%(1621)$ \\
\hline \multicolumn{5}{|l|}{ Monthly income } \\
\hline Under $\$ 500$ & $7.2 \%(71)$ & $7.5 \%(93)$ & $8.9 \%(90)$ & $7.87 \%(254)$ \\
\hline$\$ 500-\$ 2999$ & $45.3 \%(446)$ & $44.8 \%(556)$ & $45.0 \%(453)$ & $45.03 \%(1455)$ \\
\hline$\$ 3000-\$ 7499$ & $31.2 \%(307)$ & $32.1 \%(398)$ & $30.8 \%(310)$ & $31.37 \%(1015)$ \\
\hline$\$ 7500+$ & $11.8 \%(116)$ & $11.0 \%(137)$ & $10.6 \%(107)$ & $11.13 \%(360)$ \\
\hline Don't know & $4.6 \%(45)$ & $4.5 \%(56)$ & $4.7 \%(47)$ & $4.60 \%(148)$ \\
\hline \multicolumn{5}{|l|}{ Marital status } \\
\hline Single, never married & $17.0 \%(176)$ & $18.7 \%(243)$ & $20.1 \%(212)$ & $18.60 \%(631)$ \\
\hline Married & $55.7 \%(578)$ & $52.6 \%(682)$ & $51.4 \%(543)$ & $53.23 \%(1803)$ \\
\hline Separated & $2.2 \%(23)$ & $3.5 \%(46)$ & $3.1 \%(33)$ & $2.93 \%(102)$ \\
\hline Divorced & $14.2 \%(147)$ & $14.2 \%(184)$ & $16.6 \%(175)$ & $15.00 \%(506)$ \\
\hline Widowed & $3.7 \%(38)$ & $4.1 \%(53)$ & $2.7 \%(29)$ & $3.50 \%(120)$ \\
\hline Domestic partner & $7.1 \%(74)$ & $6.9 \%(89)$ & $6.1 \%(64)$ & $6.70 \%(227)$ \\
\hline Don't know & $0.1 \%(1)$ & $0.0 \%(0)$ & $0.1 \%(1)$ & $0.07 \%(2)$ \\
\hline \multicolumn{5}{|l|}{ Full-time student } \\
\hline No & $94.8 \%(934)$ & $94.6 \%(1168)$ & $94.2 \%(938)$ & $94.53 \%(3040)$ \\
\hline Yes & $5.1 \%(50)$ & $5.3 \%(65)$ & $5.5 \%(55)$ & $5.30 \%(170)$ \\
\hline Don't know & $0.1 \%(1)$ & $0.2 \%(2)$ & $0.3 \%(3)$ & $0.20 \%(6)$ \\
\hline \multicolumn{5}{|l|}{ Age } \\
\hline $\mathrm{M}(\mathrm{SD})$ & $48.91(13.20)$ & $48.49(13.70)$ & $47.63(13.62)$ & $48.34(13.51)$ \\
\hline \multicolumn{5}{|l|}{ Age range } \\
\hline Minimum & 18 & 18 & 18 & 18 \\
\hline Maximum & 86 & 86 & 86 & 86 \\
\hline
\end{tabular}

\section{Procedure}

Once individuals provided consent and completed the baseline assessment, they were given instructions on how to proceed based on their assigned group. Individuals in the control group were instructed to return to the Web site for their 6-month follow-up assessment and were told that they would receive an e-mail reminder. Those randomized to the online treatment group were immediately brought into their first online intervention (details to follow), and then received an e-mail reminder at 3 and 6 months to $\log$ in for their second and third interventions, respectively. A few days following completion of their 6 month intervention, a reminder was sent to return to the Web site to complete their follow-up assessment. Those assigned to the telephonic treatment group were told that they would be receiving a call from their coach within the next week to complete their first intervention. During each session, the coach arranged for the next telephonic coaching session (3 and 6 months respectively). A few days following completion of their 6-month intervention, a reminder was sent to return to the Web site to complete their follow-up assessment. All sessions were scheduled through the Computer Tailored Intervention (CTI)
System, and all the coaches or participants needed to do to start each session or assessment was to log in using their ID and password.

E-mail reminders were used to bring participants back for the follow-up assessment, as well as to remind individuals in the online treatment group to return for intervention sessions. Reminders for intervention sessions were automated so that if the participant did not return after the first, they would continue to receive them until the individual did return or 3 months had passed. Some participants returned for only 1 or 2 intervention sessions. However, they then received an additional series of reminders to return for the follow-up assessment. Given this, the intervention dose differed among participants and therefore was included in the multiple imputation analysis. For those in the telephonic treatment group, follow-up intervention phone calls were placed at scheduled times. If the participant was not available at the scheduled time, the coaches continued to call for a maximum of 14 calls until the participant was reached. As with the online group, a separate set of reminders (both e-mail and telephonic) were used to remind participants to complete their follow-up assessment. Again, the intervention dose differed among participants and 
Table 2. Participant Baseline Risks and Behaviors

\begin{tabular}{|c|c|c|c|c|}
\hline & $\begin{array}{l}\text { Group } 1 \\
\text { Control }\end{array}$ & $\begin{array}{c}\text { Group } 2 \\
\text { Stress Online }\end{array}$ & $\begin{array}{c}\text { Group } 3 \\
\text { Exercise Coaching }\end{array}$ & Total (1-3) \\
\hline \multicolumn{5}{|l|}{ BMI } \\
\hline Underweight & $1.7 \%(18)$ & $1.3 \%(17)$ & $2.2 \%(23)$ & $1.73 \%(58)$ \\
\hline Normal & $25.1 \%(260)$ & $23.9 \%(310)$ & $20.5 \%(217)$ & $23.17 \%(787)$ \\
\hline Overweight & $30.9 \%(320)$ & $29.0 \%(376)$ & $32.3 \%(341)$ & $30.73 \%(1037)$ \\
\hline Obese & $42.3 \%(439)$ & $45.8 \%(594)$ & $45.0 \%(476)$ & $44.37 \%(1509)$ \\
\hline \multicolumn{5}{|l|}{ Have you ever smoked? } \\
\hline Yes & $57.8 \%(599)$ & $57.5 \%(573)$ & $56.1 \%(425)$ & $57.1 \%(1597)$ \\
\hline $\begin{array}{l}\text { Depression prevention } \\
\text { reported no depression }\end{array}$ & $22.2 \%(230)$ & $17.7 \%(176)$ & $20.7 \%(157)$ & $20.2 \%(563)$ \\
\hline \multicolumn{5}{|l|}{ Stage of change } \\
\hline PC & $31.9 \%(331)$ & $32.3 \%(322)$ & $28.5 \%(216)$ & $30.9 \%(869)$ \\
\hline $\mathrm{C}$ & $33.9 \%(352)$ & $30.4 \%(303)$ & $31.2 \%(236)$ & $31.8 \%(891)$ \\
\hline PR & $24.3 \%(252)$ & $27.6 \%(275)$ & $29.6 \%(224)$ & $27.2 \%(751)$ \\
\hline A & $5.6 \%(58)$ & $5.3 \%(53)$ & $7.4 \%(56)$ & $6.1 \%(167)$ \\
\hline $\mathrm{M}$ & $4.2 \%(44)$ & $4.4 \%(44)$ & $3.3 \%(25)$ & $4.0 \%(113)$ \\
\hline \multicolumn{5}{|l|}{ Stage of change } \\
\hline \multicolumn{5}{|l|}{ Stress management } \\
\hline PC & $33.8 \%(351)$ & $32.8 \%(327)$ & $29.5 \%(223)$ & $32.0 \%(901)$ \\
\hline $\mathrm{C}$ & $25.1 \%(260)$ & $24.0 \%(239)$ & $26.3 \%(199)$ & $25.1 \%(698)$ \\
\hline PR & $20.7 \%(215)$ & $23.5 \%(234)$ & $24.3 \%(184)$ & $22.8 \%(633)$ \\
\hline A & $7.8 \%(81)$ & $8.2 \%(82)$ & $8.7 \%(66)$ & $8.2 \%(229)$ \\
\hline $\mathrm{M}$ & $12.5 \%(130)$ & $11.5 \%(115)$ & $11.2 \%(85)$ & $11.7 \%(330)$ \\
\hline
\end{tabular}

A, Action; BMI, body mass index; C, Contemplation; M, Maintenance; PC, Precontemplation; PR, Preparation.

therefore was included in the multiple imputation analysis. Individuals were able to return to the program and complete an online session, or call in to a 1-800 number to do a coaching session at any time during the 6-month study even if they had not completed previously scheduled sessions. A CTI session was able to be completed once every 2 months.

\section{Incentives}

Individuals received incentives for participation in the program. Those who completed the baseline assessment were eligible for a drawing through the survey company that assisted in recruitment. All subsequent interactions were incentivized by points that could be redeemed for gift cards. The amount of points increased at each interaction so that a participant could receive a maximum total of $\$ 75$. The incentives were: $\$ 10$ for the first intervention, $\$ 15$ for the second intervention, $\$ 20$ for the third intervention, and $\$ 30$ for the follow-up assessment completion. Individuals could still receive the $\$ 30$ follow-up assessment incentive even if they did not complete all 3 intervention time points.

\section{Interventions}

Both treatments employed CTIs to deliver tailored evidencebased sessions to the participants. The groups differed on communication method (online vs. through a telephonic coach) and the primary behavioral focus (stress management vs. exercise). Both treatment groups received a higher level of tailoring for a primary behavior (full tailoring for online, optimal TTM tailoring for coaching) and only stage-matched tailoring for the secondary behavior.

These 2 treatments were used, in part, because telephonic coaching and online CTIs represent the 2 most commonly applied modalities of scalable wellness interventions. This study was not designed, however, to compare whether telephonic coaching produced greater impact than online CTIs or whether exercise as the primary behavior was more effective than stress management, because treatment modality and primary behaviors were confounded rather than controlled for.

Levels of tailoring. Full tailoring (stress online) employs assessments and tailoring on the 14 TTM variables that can produce progress at each stage of change. The first session provides normative feedback (compared to peers in same stage who were successful). Follow-up sessions provided normative and ipsative (compared to self) feedback on each of the relevant TTM variables. The program first assesses an individual on a TTM variable, like the pros of changing, compares the score to normative and ipsative databases and then immediately provides feedback (eg, overutilizing, underutilizing, or utilizing enough compared to successful peers).

Optimal tailoring (exercise coaching) uses the same approach of assessment and tailoring, but only on the 3 to 5 TTM variables that account for the most progress at each stage of change. This more recent approach is called optimal because it is designed to maximize change while limiting tailoring demands on participants and coaches. ${ }^{27}$ The secondary behaviors in each intervention received stage-matched tailoring, which only tailors on stage of change by giving guidance on the one or two steps that could lead to the next stage. This type of protocol can simultaneously treat multiple behaviors while reducing the treatment demands on participants and providers. This protocol has been found to be effective in 
simultaneously changing multiple behaviors in both adults $^{28}$ and adolescents. ${ }^{29,30}$

Exercise coaching intervention. The exercise coaching group received up to 3 proactive telephone sessions at 0,3 , and 6 months via outreach by a trained health coach. The same coach did not necessarily call the participant each time. Coaches used the CTIs to guide all sessions with optimal TTM tailoring for exercise and stage tailoring for stress management, the secondary target. Following a calendar of scheduled calls, the coach would call the participant at the designated time and log in to the CTI using the participant's ID and a password specified for the coach. The CTI then leads the coach through a series of assessment questions and tailored feedback, based on stage of change and TTM variables, to guide their participants to the next stage of change. As an example, the coach would first be directed to assess the participant's stage of change for exercise. Once the participant answered, the coach would input that response by clicking on the correct button, and then the next screen would give bulleted type feedback that the coach could share with the participant, and also tips for the coach on how to interact with them. For example, if a participant was in the Precontemplation Stage of Change when the pros of changing are an important construct to focus on, and the participant was not recognizing the pros enough to make progress and had not improved since the last time, the coach would have a screen that stated:

\section{Pros: NEGATIVE}

- You're still not thinking enough about the pros of regular exercise. In fact, you're not any more aware of the benefits than you were the last time we talked.

- Let's come up with some pros for you to focus on.

Some pros:

- Makes your heart stronger and more efficient

- Helps you maintain a healthy weight

- Improves your flexibility, muscle strength, and endurance

- Helps you feel more self-confident.

- Which pro is most important to you?

The coach would use these points to inform their interaction with the participant. The session would then continue when the coach was ready to move on to the next set of questions, and the process would continue through the relevant constructs for that participant at that time. Each question, feedback, and instructions to the coaches were tailored to the specific tailored, evidence-based procedure for that person. The coaches assigned to this project were Healthways Coaches, who received additional training by the Pro-Change e-Learning Program (basic TTM) and by the Pro-Change staff. Each telephone session lasted about 20 minutes.

Stress management online intervention. The stress management online group received a self-directed Webbased intervention with full TTM tailoring for stress management and stage-based tailoring for exercise. The decision rules, algorithms, and navigation through the CTI were similar to the coaching system. However, all content was participant facing (the participant read it off the screen), and spoke directly to them rather than through a coach. All aspects of the screen were tailored to the individual's needs based on previous research, including the questions, images, and feedback. The program was self-directed and was very user friendly. A sample onscreen message, for someone in the Precontemplation stage who was not recognizing the pros of stress management enough and had not made progress since the last session is listed below.

Effective stress management takes some work, but it pays off in so many ways!

You're still not aware of the pros (benefits) of using healthy strategies.

Once you know the pros, you'll find it easier to think more seriously about beginning to manage your stress. Here are just a few of the benefits you could enjoy:

- Managing your stress can give you more energy.

- Using healthy strategies may help you solve the problems causing your stress.

- Many healthy strategies, such as exercise and relaxation, are good for your heart and your mind.

- Using healthy strategies sets a good example.

- You may feel more confident.

As you pay more attention to the benefits, you'll start thinking more about making effective stress management a way of life.

Can you think of one pro that is important to you? Type it in the space below.

The participant was led through a series of assessments that were tailored to his or her individual evidence based needs at the time, and received feedback paragraphs similar to the one above interspaced within the assessments.

\section{Measures}

All participants were administered the assessments online at baseline and after 6 months for evaluation purposes. The baseline and follow-up assessments incorporated the following measures used in this study:

Demographics. Age, sex, ethnicity, income, marital and work status were assessed.

Health behaviors/risks. The assessment from the Health Risk Intervention ${ }^{31}$ was employed to assesses risk status and stage of change for 9 health risk behaviors: smoking, physical activity, healthy diet (low-fat and calorie control), fruits and vegetables, effective stress management, depression prevention, alcohol consumption, adherence to antihypertensive and cholesterol medication. ${ }^{31}$ This study focused on exercise, stress, healthy diet, depression prevention, and overall behavior risk, measured by the total number of risk factors (0-9).

Well-being. The Individual Well-being Scores (IWBS) assessment consists of 53 items representing 6 domains of well-being: Life Evaluation, Physical Health, Emotional Health, Work Environment, Healthy Behaviors, and Basic Access. ${ }^{20}$ These well-being domains are consistent with those used in the Gallup-Healthways Well-Being Index, a nationwide community-based survey of well-being. ${ }^{32}$ The Life Evaluation domain is based on combined responses from the Cantril Self-Anchoring Scale, ${ }^{33}$ for which participants are asked to rate their present and future life from 0 (worst possible life) to 10 (best possible life). This measure can be 
scored continuously or categorically as Thriving (7 or higher for present life and 8 or higher for future life), Suffering (4 or below for both present and future life), and Struggling (intermediate scores between Thriving and Suffering).

The primary outcome measures of interest were stage of change for health risk behaviors, where success was defined as entering the Action or Maintenance stage, and well-being (overall and individual domains), which was measured on a continuous scale from 0 to 100, with the exception of Life Evaluation, which could be scored categorically, as indicated. Because some participants had already reached Action or Maintenance on a behavior by the baseline assessment, those individuals were excluded from analyses of outcomes for which they had achieved the criteria. The 2 treatment groups also were assessed at each treatment session on TTM variables that were used for tailoring their treatment. Figure 1 presents the participation rates at each assessment and treatment contact. All procedures were approved by the ProChange Behavior Systems, Inc. Institutional Review Board.

\section{Statistical analysis}

Multiple imputation (MI) ${ }^{34,35}$ was used to estimate missing data for the 6-month follow-up. MI, which Schafer and Graham $^{36}$ describe as being the state of the art for missing value replacement, uses a simulation technique to replace each missing value with a set of plausible values, resulting in multiple complete data sets that differ only in the imputed values. This is the approach used with a series of highly cited population-based multiple behavior change RCTs, $22,23,31$ including a healthy weight management project with a large percentage of missing data. ${ }^{24}$ For these studies, typically 10 data sets were imputed to provide an optimal level of reliability and valid estimates for the missing data. For the current study,
10 data sets were imputed using Multivariate Imputation by Chained Equations, ${ }^{37}$ which is implemented by $\mathrm{R}$ package 'mice' version 1.16. Tests of significance take into account the uncertainty introduced by the missing data and the uncertainty in the imputed values, and so yield more accurate estimates of confidence intervals and significance levels than other missing data approaches. ${ }^{34}$ The imputation model included baseline variables (sex, ethnicity, income, education, employment status, marital status, total behavior risk status, and intervention dose) that met 2 criteria. First, they needed to meet criteria for variables that produce the best estimates of missing data, such as variables with a small number of categories, such as sex. Including stages of change for all measured risk behaviors, for example, would not be as effective as including 2 categories of stage being in pre-action stages (Precontemplation, Contemplation, and Preparation) or in post-action (Action and Maintenance) stages. Variables also were selected that, from our research, are related to outcomes being imputed and whether individual data was missing. Research has demonstrated that the more baseline variables used for imputing, the more accurate are the results. ${ }^{38}$ Statistical significance of the pooled results was evaluated using a $t$ test and degrees of freedom. Logistic regression analyses of health behaviors measured the stage progression of individuals in pre-action stages at baseline for each behavior; progression to the Action or Maintenance stage defined treatment success. Individuals in Action or Maintenance at baseline for a particular behavior were necessarily excluded from corresponding analyses that focused on movement to non-risk status as they were already at criteria.

Additional analyses used a complete case approach, omitting participants with missing follow-up data to assess for replication of outcomes without imputing missing data. Because these results did not materially differ from those of the MI analyses, only MI results are included.
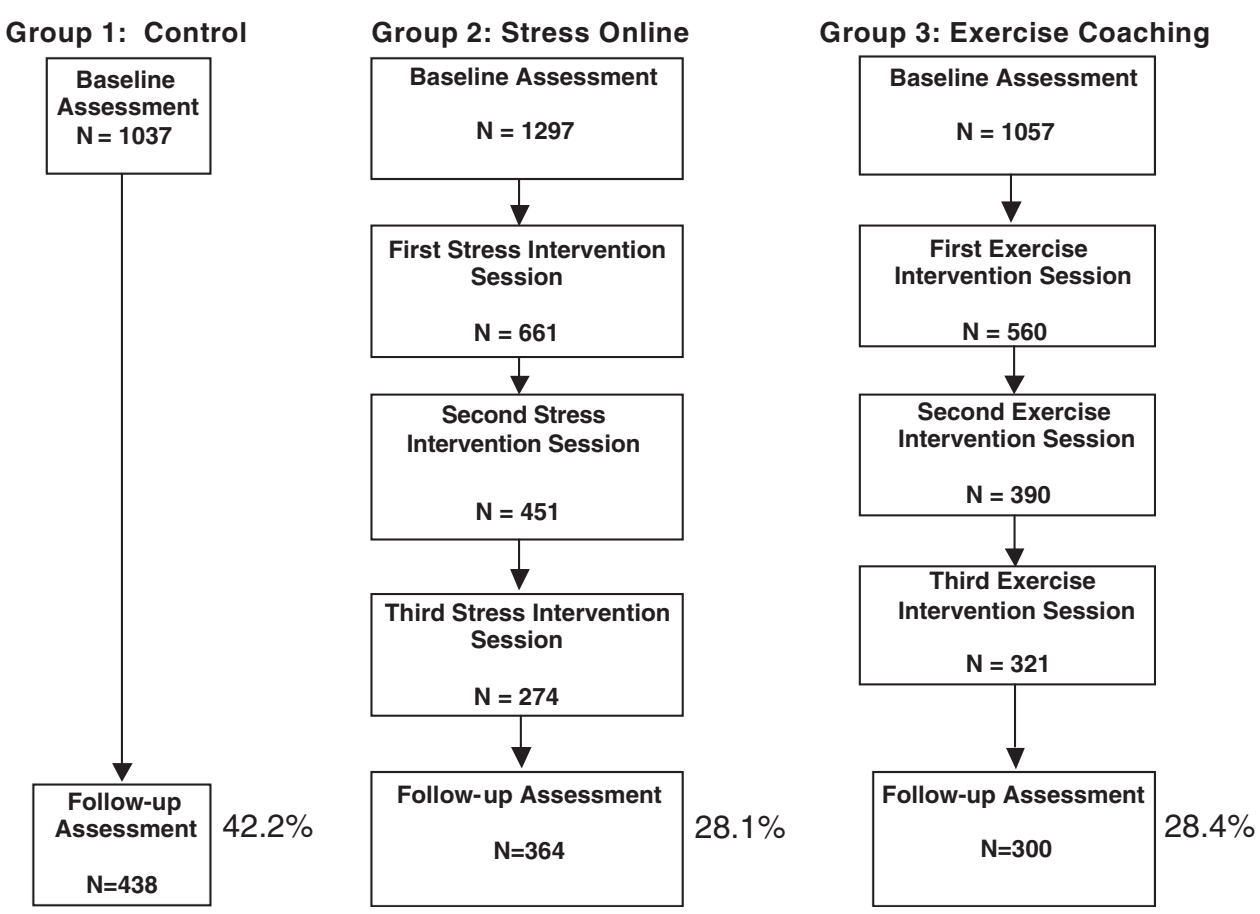

FIG. 1. Overall trial retention. 
Table 3. Health Behavior Risk Change in Control and Intervention Groups

\begin{tabular}{|c|c|c|c|c|c|c|c|c|c|c|}
\hline \multirow[b]{2}{*}{ Health Risk Behavior } & \multicolumn{2}{|c|}{ Control } & \multicolumn{2}{|c|}{ Online } & \multicolumn{2}{|c|}{ Coaching } & \multicolumn{2}{|c|}{$\begin{array}{c}\text { Online vs. } \\
\text { Control }\end{array}$} & \multicolumn{2}{|c|}{$\begin{array}{l}\text { Coaching ws. } \\
\text { Control }\end{array}$} \\
\hline & Baseline $\mathrm{n}$ & \% Progress & Baseline $\mathrm{n}$ & $\%$ Progress & Baseline $\mathrm{n}$ & \% Progress & $d f$ & $t$ & $d f$ & $t$ \\
\hline Exercise & 935 & 37.3 & 1167 & 46.6 & 955 & 57.3 & 23.21 & $2.55^{*}$ & 16.31 & $4.34^{* * *}$ \\
\hline Stress management & 826 & 53.1 & 1038 & 64.7 & 844 & 74.9 & 52.92 & $3.89^{* * *}$ & 17.19 & $4.83^{* * *}$ \\
\hline Healthy eating & 813 & 21.1 & 1033 & 26.4 & 844 & 30.7 & 44.79 & 1.9 & 21.92 & $2.85^{* *}$ \\
\hline Depression & 548 & 58.9 & 709 & 63.7 & 568 & 67.3 & 105.37 & 1.35 & 68.74 & $2.19^{*}$ \\
\hline Overall risks & 1037 & - & 1297 & - & 1057 & - & 37.64 & $-3.30^{* * *}$ & 21.39 & $-5.28^{* * *}$ \\
\hline
\end{tabular}

${ }^{*} P<0.05 ;{ }^{* *} P<0.01 ;{ }^{* * *} P<0.001$.

\section{Results}

\section{Health risk behavior outcomes}

Exercise. At 6-month follow-up, participants in the coaching group with exercise as the primary target behavior $(57.3 \%)$ were more likely to progress to Action or Maintenance compared to the control group (37.8\%). Those in the online group with exercise as the secondary behavior to stress $(46.6 \%)$ were also significantly more likely to achieve treatment success than the control group.

Stress management. A significantly greater percentage of the coaching group reached the Action or Maintenance phase for stress management at 6 months compared to the control group, (74.9\% vs. 53.1\%). Significantly more participants in the online group $(64.7 \%)$ also progressed to criteria than the control group.

Additional behavioral risks. Significant differences between the coaching and control groups were found for overall change in health behavior risk (average risk reduction of 1.18 vs. 0.49 ). Those in the online group also reported a greater reduction in number of behavior risks ( 0.82 average risk reduction) compared to the control group. With respect to specific behaviors, for healthy eating, those in the coaching group were significantly more likely to progress to Action or Maintenance at 6 months compared to the control group (30.7\% vs. $21.1 \%$ ). Participants in the coaching group $(67.3 \%)$ were more likely to progress to Action or Maintenance for depression prevention at 6 months compared to the control group $(58.9 \%)$. Table 3 displays summary results for all health risk behavior outcomes.

\section{Well-being outcomes}

Well-being domains. Table 4 shows the results of $t$ tests examining the effects of the interventions on well-being domains. The pattern across overall well-being and its 6 domain scores showed the coaching group produced the highest improvement on each well-being score, followed by the online and control groups. Significant differences were found between the coaching and control group and the online and control group on overall well-being and on 4 of the 6 domain scores: Emotional Health; Physical Health; Life Evaluation; and Healthy Behaviors (Fig. 2).

Life evaluation categories. Figure 3 , shows that $62.3 \%$ of participants in the coaching group were classified as Thriving at 6-month follow-up, compared to the $55.0 \%$ and $50.1 \%$ in the online and control groups, respectively.

Among those classified as Suffering or Struggling for Life Evaluation at baseline, participants in the coaching group progressed to a Thriving status at a higher rate compared to the control group, $t(77.29)=5.35, P<0.001(49.3 \%$ vs. $31.4 \%)$. Similarly, a significantly greater percentage of those in the online group (41.4\%) progressed to Thriving compared to the control group, $t(289.13)=3.6, P<0.001$.

\section{Discussion}

This study was the first to demonstrate that 2 wellness programs could produce significant improvement on both multiple health risk behaviors and multiple domains of well-being. The results indicate that compared to the control group, both the telephonic coaching and the online CTI were able to produce significantly greater changes on the primary target behavior that received more intensive treatment as well as the secondary behavior that received stage-matched help only. Comparing the average numbers of behaviors changed per participant, both the telephonic coaching ( $\mathrm{M}=1.2$ behaviors) and the online CTI $(\mathrm{M}=0.8$ behaviors) were able to produce significantly greater changes than the control group ( $M=0.5$ behaviors). The highest previously reported treatment outcome was an average reduction of 0.77 behaviors for both treated and untreated behaviors, while the control group had 0.51 behaviors changed. ${ }^{24}$ Further, both treatments were able to produce significantly greater enhancements than the control group on overall well-being and 4 domains of well-being (emotional health, physical health, life evaluation, and healthy behaviors).

The outcome with the largest magnitude of change in this study was the increase in the percentage of participants who were Thriving. The magnitude of change was greater in the coaching group than the online group; this pattern of results mirrors what was found for changes in health risk behaviors. Therefore, the more behavior changed per condition, the greater the percentage of participants who progressed to Thriving, which suggests that the reduction in health risk behaviors may have produced an increase in the Thriving condition. This hypothesis will need to be tested in treatment mediation analyses, which are under way.

The above-stated hypothesis assumes that a well-being construct like Thriving can be enhanced directly by successful changes in regular exercise and effective stress management. An alternative hypothesis is that health risk behaviors and 


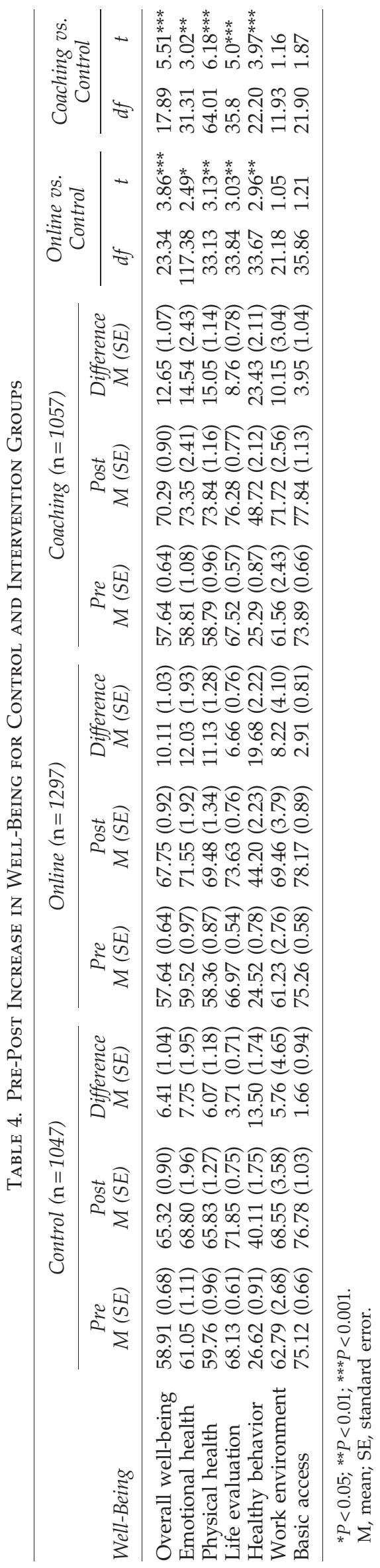

well-being domains change together. Coaction of 2 behaviors is defined by the increased probability that individuals who progress to effective action on one behavior often progress to effective action on a second behavior in concert. We have found that coaction occurs much more in treatment groups than in control groups, suggesting that the phenomenon is a direct result of the intervention, as opposed to naturally occurring. ${ }^{24,29,31}$

Confidence in the results of this study is supported, in part, by similarities of behavior outcomes to what has been found in other studies with higher retention rates. The results with the 2 treatment conditions for the percentage taking effective action with stress management were $64.7 \%$ for the online group and $74.9 \%$ for the coaching group. For exercise, results were $46.6 \%$ for the Internet condition and $57.3 \%$ for coaching. The magnitude of these results was in the expected range. They are comparable to the $60 \%$ progression to Action or Maintenance among individuals receiving CTIs for stress by mail and telephone in a national sample, ${ }^{26}$ as well as the progression of $70 \%$ for stress and $48 \%$ for exercise in an employee sample interacting with CTIs on the Internet. ${ }^{31}$ The range of outcomes with stress and exercise are somewhat higher than with previous populations, but this may be due to the fact that this population had more risks at baseline and, therefore, more opportunity for change. The entire sample was at risk with both stress and exercise, about $75 \%$ were obese or overweight, the majority were suffering and struggling, and they had much lower than normal scores on emotional health. We have found that the more stressed individuals are, the more likely they are to take significant action to reduce the aversive characteristics of stress. ${ }^{26,31}$

As anticipated from previous research, the greater the number of health risks eliminated per participant in each treatment condition, the greater the increases in personal well-being. ${ }^{20}$ From this past research on well-being domains, we would not expect the coaching online treatment to outperform the control conditions on Basic Access or Work Environment domains, which are more under community and company influences rather than individual control. ${ }^{20}$ The lack of significant changes in Basic Access and the Work Environment provide discriminative validity to the results.

Comparing outcomes across the 4 Well-Being domains that demonstrated significant change, the largest improvements were in Life Evaluation (Thriving) and Emotional Health, and the smallest changes were in overall well-being and the Healthy Behavior domain. Although it seems counterintuitive that the Healthy Behavior domain did not show a large change in magnitude as a result of the interventions, this was not unexpected given that items in this domain focus primarily on healthy diet, which was not the focus of intervention tailoring.

Although this study was not designed in such a way as to allow for a direct comparison between the 2 treatment groups, we did consider what might account for the pattern in which the exercise coaching group consistently produced health behavior and well-being changes that, on an absolute basis, were larger than the stress management online group. One hypothesis is that coaching from a caring professional may be more effective than an individualized and interactive intervention delivered on the impersonal Internet. This hypothesis is challenged, in part, by the fact that we rarely have found that a coach alone or adding a coach to 


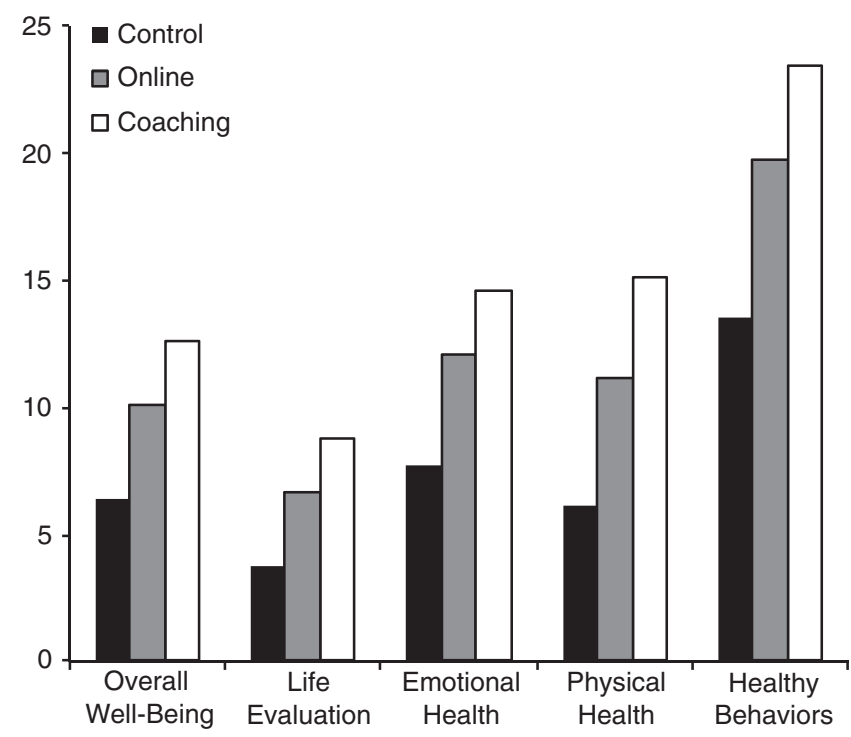

FIG. 2. Individual Well-Being Score mean difference scores.

CTIs has outperformed the CTIs alone. ${ }^{31,39,40}$ We have found, however, that adding tailored CTIs to the best practice of face-to-face counselors has improved outcomes compared to counselors alone. ${ }^{41}$ However, none of these prior studies have evaluated well-being, and person-to-person coaching may add value with particular well-being domains.

Questions must be raised as to why the control group improved as much as they did. This is a finding that has emerged consistently in all of our population based intervention trials ${ }^{22-24,26,28,31}$ in which control groups improved at rates considerably higher than secular trends. One likely factor is the phenomenon called "mere measurement" in which simply measuring people's behavior over time results in significant improvement on the measured behaviors. ${ }^{42}$ Another likely contributor is based on our analysis of how proactive population trials differ from traditional reactive clinical trials. In traditional RCTs, participants are seeking help and intend to be treated. The intervention groups receive the types of treatments they intend to receive, but the control group receives less than they intended. In population trials in which we proactively reach out to people to offer help, the people were not seeking help and did not intend to be treated. All groups receive more than they intended, including the control group. Effective behavior change treatments generally include: the 5 As (Ask, Assess, Advise, Assist, and Arrange follow-up). ${ }^{43}$ In this study, the control group received each of these treatment components except Advise and Assist, which may have contributed to their improvements.

Another factor that may have contributed to the improvements in the control group is stress adaptation. We have found that the more stressed individuals are in control groups, the more likely they are to take significant action to reduce the stress, similar to what occurs in treatment groups. ${ }^{26,31}$ This stress adaptation is likely to affect other health behaviors as well as aspects of well-being. The fact that the control group improved as much as it did makes it harder for treatment groups to outperform the controls and, in that sense, is a limitation that loads against finding significant treatment effects. In spite of this limitation, significant effects were found consistently for both treatment conditions on all treated risk behaviors and on well-being.

\section{Limitations}

The primary limitation of this study is the low retention rates in the treatment groups; the larger retention rate in the control group may have biased the outcomes in favor of the treatment conditions. To ensure that this limitation did not affect our conclusions, we employed MI analyses to detect such biases. If substantial bias existed, the complete case analyses would have been notably different from the MI results, but the patterns were essentially the same. The one exception was that there were more statistically significant results with MI because of the inclusion of all subjects, producing greater statistical power. The MI analyses are methodologically the best approach for dealing with missing data because they include all available data on all participants that we intended to treat. Quantitative studies, both with real data and simulated data, have demonstrated that when

\section{Baseline}

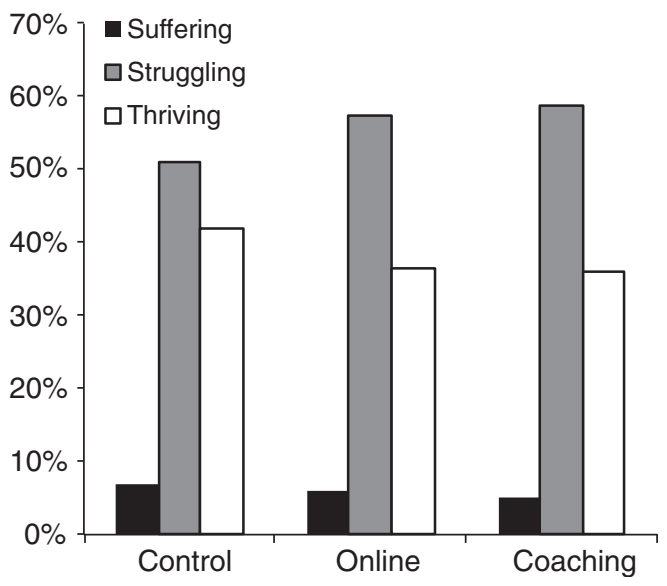

6 Month Follow-up

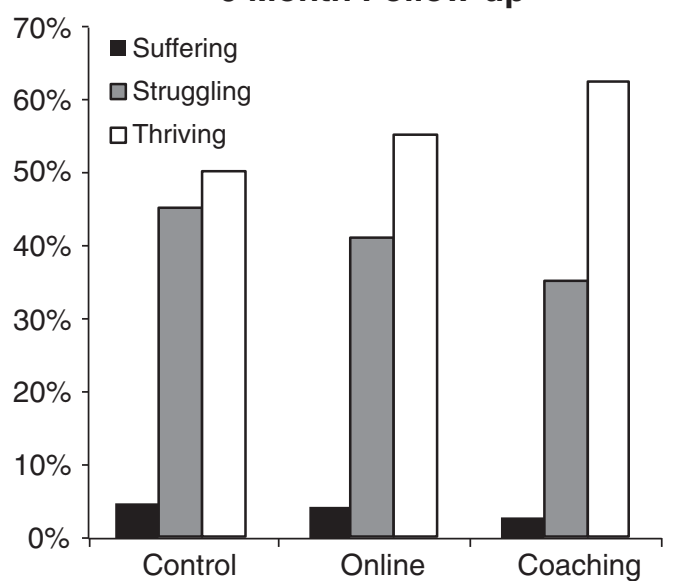

FIG. 3. Percent of participants in life evaluation categories. 
using a large enough number of imputations, such as the 10 used in this study, and including variables related to whether data were missing as well as the outcomes of interest, acceptable estimates of missing data can be generated even when $50 \%$ or more of the data are missing. ${ }^{38}$ As indicated earlier, similar analytic procedures have been used in our highly cited population-based RCTs, ${ }^{22,23}$ including a healthy weight management project with about $50 \%$ missing data in the treatment group and significantly less $(33 \%)$ in the control group. $^{24}$

The low retention rates may stem from the fact that the survey sampling company typically conducts research with only 1-time assessments or surveys. The current protocol was an exception to the typical for these participants, although the expectation was explained in all project materials. Furthermore, the assessment was lengthy. To set expectations appropriately, our introductory communication and informed consent forms explained that our project was demanding, longitudinal, and might involve participation in interventions; however, other methods such as guaranteed incentives upon completion may have improved retention. Future work should replicate this trial with improved means to produce higher retention rates.

Another important limitation of this study is the lack of a long-term follow-up. Based on previous research with stress and exercise, we would predict stable treatment effects from the 6-month assessment to longer term follow-up. This is the treatment trajectory we have consistently found with the effective stress management treatment, which produced $60 \%-70 \%$ success at 6 months that was maintained at 12 and 24 months. ${ }^{26,31}$ Stable treatment trajectories also have been found with our exercise treatments with 6-month success rates being stable at 12,18 , and 24 months. ${ }^{24,28,31}$ These predictions of stable treatment effects will need to be tested in studies with longer term follow-up.

\section{Conclusion}

This study demonstrated that TTM tailored interventions, delivered by phone or Web, can simultaneously reduce multiple health risk behaviors and enhance multiple domains of well-being. These results provide important support for tailored behavior change interventions as a means to not only reduce health risk factors, but also to enhance the overall well-being of participants. Future research, including field studies, need to be conducted to assess replication of results with longer term follow-up and higher retention rates.

\section{Acknowledgments}

This research was supported by funding from Healthways, Inc.

\section{Disclosure Statement}

James O. Prochaska, Ph.D., is a consultant to Pro-Change Behavior Systems, Inc. and Healthways, Inc. Drs. Evers, Castle, and Johnson are employees of Pro-Change Behavior Systems, Inc. Pro-Change is currently licensing the programs described herein to Healthways. Janice Prochaska, Ph.D., is the President and Chief Executive Officer of Pro-Change Behavior Systems, Inc. Drs. Rula, Coberley, and Pope are employees of Healthways, which is currently marketing the programs described herein.

\section{References}

1. US Department of Health and Human Services. Healthy People 2020 Framework. http://www.healthypeople.gov/ hp2020/Objectives/framework.aspx. Accessed August 27, 2010.

2. World Health Organization. Preamble to the Constitution of the World Health Organization as adopted by the International Health Conference, New York, 19-22 June 1946, and entered into force on 7 April 1948.

3. Achat H, Kawachi I, Spiro A, III, DeMolles DA, Sparrow D. Optimism and depression as predictors of physical and mental health functioning: The Normative Aging Study. Ann Behav Med 2000;22:127-130.

4. Murrell SA, Salsman NL, Meeks S. Educational attainment, positive psychological mediators, and resources for health and vitality in older adults. J Aging Health 2003;15:591-615.

5. Ostir GV, Markides KS, Black SA, Goodwin JS. Emotional well-being predicts subsequent functional independence and survival. J Am Geriatr Soc 2000;48, 473-478.

6. Loeppke R, Taitel M, Haufle V, Parry T, Kessler RC, Jinnett $\mathrm{K}$. Health and productivity as a business strategy: A multiemployer study. J Occup Environ Med 2009;51:411-428.

7. Murray C, Lopez A. Alternative projections of mortality and disability by cause 1999-2020: Global Burden of Disease Study. Lancet 1997;349:1498-1504.

8. Unutzer J, Patrick D, Diehr Pl, Simon G, Grembowski D, Katon W. Quality adjusted life years in older adults with depressive symptoms and chronic medical disorders. Int Psychogeriatr 2000;12:15-33.

9. Lyubomirsky S, King L, Diener E. The benefits of frequent positive affect: Does happiness lead to success? Psychol Bull 2005;131:803-855.

10. Stampfer MJ, Hu FB, Manson JE, Rimm EB, Willett WC. Primary prevention of coronary heart disease in women through diet and lifestyle. N Engl J Med 2000;343:16-22.

11. Schultz AB, Edington DW. Employee health and presenteeism: A systematic review. J Occup Rehabil 2007;17:547-579.

12. Spector PE. Job Satisfaction: Application, Assessment, Cause, and Consequences. Thousand Oaks, CA: Sage, 1997.

13. Gilboa S, Shirom A, Fried Y, Cooper C. A meta-analysis of work demand stressors and job performance: Examining main and moderating effects. Personnel Psychol 2008;61;227-271.

14. Veenhoven R. World database of happiness: Correlates of happiness: 7837 findings from 603 studies in 69 nations 1911-1994 (3 vols.) Rotterdam, the Netherlands: RISBO, 1994.

15. Mackenbach JP, Howden-Chapman P. New perspectives on socioeconomic inequalities in health. Perspect Biol Med 2003;46;428-444.

16. Diener E, Seligman MEP. Beyond money: Toward an economy of well-being. Psychol Sci Public Interest 2004:5;1-31.

17. Peterson C, Park N, Seligman MEP. Orientations to happiness and life satisfaction: The full life versus the empty life. J Happiness Stud 2005:6; 25-41.

18. Fredrickson BL, Cohn MA, Coffey KA, Pek J, Finkel SM. Open hearts build lives: Positive emotions, induced through loving-kindness meditation, build consequential personal resources. J Pers Soc Psychol 2008:95;1045-1-62.

19. Merrill RM, Aldana SG, Pope JE, et al. Evaluation of a bestpractice worksite wellness program in a small-employer setting using selected well-being indices. J Occup Environ Med. 2011:53;448-454. 
20. Prochaska JO. Productive well-being. Presented at Integrated Care Summit, Oct 16, 2010, Washington DC.

21. Jones H, Edwards L, Vallis TM, et al. Changes in diabetes self-care behaviors make a difference in glycemic control: the diabetes stages of change (DISC) study. Diabetes Care 2003:26;732-737.

22. Prochaska JO, Velicer WF, Rossi JS, et al. Multiple risk expert systems interventions: Impact of simultaneous stagematched expert system interventions for smoking, high-fat diet, and sun exposure in a population of parents. Health Psychol 2004:23;503-516.

23. Prochaska JO, Velicer WF, Redding CA, et al. (2005). Stagebased expert systems to guide a population of primary care patients to quit smoking, eat healthier, prevent skin cancer, and receive regular mammograms. Prev Med 2005:41;406-416.

24. Johnson SS, Paiva AL, Cummins CO, et al. Transtheoretical model-based multiple behavior intervention for weight management: Effectiveness on a population basis. Prev Med 2008:46;238-246.

25. Reed GR, Velicer WF, Prochaska JO, Rossi JS, Marcus BH. What makes a good staging algorithm: Examples from regular exercise. Am J Health Promot 1997:12;57-66.

26. Evers KE, Prochaska JO, Johnson JL, Mauriello LM, Padula JA, Prochaska JM. A randomized clinical trial of a population-and Transtheoretical Model based stress management intervention. Health Psychol 2006:25; 21-529.

27. Noar SM, Benac CN, Harris MS. Does tailoring matter? Meta-analytic review of tailored print health behavior change interventions. Psychol Bull 2007;133:673-693.

28. Johnson SS, Driskell MM, Johnson J., et al. Transtheoretical model intervention for adherence to lipid-lowering drugs. Dis Manag 2006:9;102-114.

29. Mauriello LM, Ciavatta MM, Paiva AL, et al. Results of a multi-media multiple behavior obesity prevention program for adolescents. Prev Med 2010;51:451-456.

30. Redding CA, Prochaska JO, Paiva A, et al. Baseline stage, severity, and effort effects differentiate stable smokers from maintainers and relapsers. Subst Use Misuse 2011;46:1664-1674.

31. Prochaska JO, Butterworth $S$, Redding CA, et al. Initial efficacy of MI, TTM tailoring and HRI's with multiple behaviors for employee health promotion. Prev Med 2008: 46;226-231.

32. Gallup, Inc. Gallup-Healthways Well-Being Index: Methodology Report for Indexes. Available at: http://www.well-beingindex .com/files/Gallup-Healthways\%20Index\%20Methodology\% 20Report\%20FINAL\%203-25-08.pdf. Accessed May 26, 2011
33. Cantril H. The Pattern of Human Concerns. New Brunswick, NJ: Rutgers University Press, 1965.

34. Rubin DB. Multiple Imputation for Nonresponse in Surveys. New York, NY: John Wiley \& Sons Inc., 1987.

35. Harel O, Zhou XU. Multiple imputation: Review of theory, implementation and software. Stat Med 2007:26;3057-3077.

36. Schafer JL, Graham JW. Missing data: Our view of the state of the art. Psychol Meth 2002:7;147-177.

37. Van Buuren S, Oudshoorn CGM. Multivariate Imputation by chained equations, V1.1.0 for R. Available at: http:// web.inter.nl.net/users/S.van. Buuren/mi/hmtl/mice.htm. Accessed February 25, 2004.

38. Collins LM, Schafer JL, Kam CM. A comparison of inclusive and restrictive strategies in modern missing data procedures. Psychol Meth 2001:6;330-351.

39. Prochaska JO, DiClemente CC, Velicer WF, Rossi JS. Standardized, individualized, interactive and personalized selfhelp programs for smoking cessation. Health Psychol 1993:12;399-405.

40. Prochaska JO, Velicer WF, Fava J, et al. Counselor and stimulus control enhancements of a stage matched expert system for smokers in a managed care setting. Prev Med 2001:32;23-32.

41. Lawrence T, Aveyard P, Evans O, Cheng KK. A cluster randomized controlled trial of smoking cessation in pregnant women comparing interventions based on the transtheoretical (stages of change) model to standard care. Tob Control 2003:12;168-177.

42. Godin G, Sheeran P, Conner M, Delage G, Germain M, Belanger-Gravel A, Naccahe $\mathrm{H}$. Which survey questions change behavior? Randomized controlled trial of mere measurement interventions. Health Psychol 2010:29; 636644.

43. Whitlock EP, Orleans CT, Pender N, Allan J. Evaluating primary care behavioral counseling interventions. An evidence-based approach. Am J Prev Med 2002;22:267-284.

Address correspondence to: Kerry E. Evers, Ph.D. Pro-Change Behavior Systems, Inc. PO Box 755 West Kingston, RI 02892

E-mail: kevers@prochange.com 